\title{
Editorial
}

\section{A Note from Management and Organization Review: A Warm Welcome and a New Volume}

\author{
Anne S. Tsui \\ Arizona State University, USA
}

It is with great pleasure that we open the sixth volume of Management and Organization Review. We have successfully published five volumes and owe a great deal of gratitude to the authors, reviewers, and editors who made each issue possible. This year, 2010, will be the first year that ISI tracks citations to MOR articles, and we encourage you, our readers, to help us introduce $M O R$ to all those who are conducting or interested in management research on China.

\section{THANK YOU AND WELCOME TO THE EDITORIAL BOARDS}

We have a few changes to the MOR boards for 2010 and wish to take a moment to welcome and acknowledge the contributions of these individuals.

We have appreciated the guidance Oded Shenkar provided as a Senior Editor and Justin Tan as a Consulting Editor. Although they are stepping down from these roles, we are grateful that they will remain active $M O R$ participants as an Editorial Advisory Board member and Editorial Review Board member, respectively.

Among the editors, we warmly welcome Yadong Luo as a returning Senior Editor, Zhi-Xue Zhang as the new Chinese Abstract Editor, and Eric Tsang and Anthea Yan Zhang as new Consulting Editors. We are also delighted for Consulting Editor Patrick Wright to join the Senior Editor team.

We are very pleased to add Roy Yong Joo Chua, Scott Kennedy, Haiyang Li, Xiaowei Rose Luo, Heli Wang, and Dean Xu, all of whom previously served in an ad hoc capacity, as new Editorial Review Board members.

To those changing or entering new roles as well as those continuing their service to $M O R$, we extend our most sincere gratitude for helping shape $M O R$ into the 
go-to resource for high-caliber Chinese management research. As we start a new volume, we thank you all - our readers, authors, editors, reviewers, sponsors, and publishers!

\section{IN THIS ISSUE: ARTICLES SPANNING ASIA AND CHINA}

In this first issue of Volume 6, we present six thoughtful and exciting articles that represent the best comparative analysis and Chinese management research that we continue to publish in MOR. The first three articles analyse social and psychological issues of management in the broader Asian context while the next three articles examine management phenomena on mainland China. The first article, by Chai and Rhee, integrates a culture-embedded rational model of action and social network model of structure and enriches these with the idea of Confucian capitalism and the 'East Asian Model' of the firm. Through this integrative approach, the authors explain why network closure has been a more powerful source of productivity in East Asia than the West. The second article, by Kim, Weber, Leung, and Muramoto, compares East Asians' evaluation of preferences for task and maintenance inputs in reward allocation decisions and how these evaluations influence fairness judgments. Results show significant differences among Japanese, South Korean, and Hong Kong employees in input preferences and in fairness evaluations. Lin and Peng examine the relationship of individual level organizational citizenship behaviour to organizational performance in a sample of sales teams in Taiwan. Group cohesion and collective efficacy fully mediate this relationship. Li, Guo, $\mathrm{Yi}$, and Liu discovered a curvilinear relationship of ownership concentration to product innovation, mediated by the organization's learning orientation. Both studies provide important theoretical insight with significant managerial implications. Sun, Wright, and Mellahi offer a process model to explain the dynamic interaction between entrepreneurs and local politicians, the factors influencing the relative bargaining position of the two parties, and subsequent outcomes of the alliance. In the final article, Zhang and Keh analyse the role of two governance mechanisms, contracts and guanxi, in inter-organizational exchanges as a function of ownership form. They theorize that the importance of guanxi will decline with progress in market transition. Like the articles by Sun et al. and by Chai and Rhee, this article offers novel and institutionally-grounded logic on the behaviour of Chinese managers and firms.

Finally, this issue also publishes two commentaries debating the question of data quality, completeness, and accurate inference. This exchange suggests that our readers are carefully reading the work published in $M O R$ and are willing to challenge the authors' assumptions and research methods. This reflects a genuine desire to achieve high-quality research and is a signal that $M O R$ has a vibrant scholarly community desiring the pursuit of good science. We look forward to a new volume of path-breaking research in MOR in this year of the 'tiger'. 


\section{A NOTE ON THE GOVER IMAGE}

The cover image of this issue, titled 'Four Tiger Faces'《四象》, by artist Yusi Lin (林于思), is to commemorate the Chinese New Year, which is the Year of the Tiger. The tiger, known as the king of the forest, is a very popular symbolic image in Chinese culture. It means power, courage, and vigour. During the New Year, Chinese people like to wish each other vigour and power like the tiger (虎虎生威).

According to Mr. Lin, 'the painting shows four tiger expressions representing the four different images of China in the world today - sincere, awkward, unpredictable, and strong'.

Mr. Lin is a native of southern China, born in the Guangdong province. $\mathrm{He}$ received both a Bachelor's and Master's degree in Chinese painting from the Guangzhou Academy of Fine Arts. He is considered one of the most creative and accomplished young artists in China. He is a member of the China Artists Association and the Guangzhou Artists Association. His paintings have won many awards in China and were exhibited in the Autumn Arts Festival in France in 2007. 